\title{
On positive solutions of nonlinear elliptic equations involving concave and critical nonlinearities
}

\author{
by
}

\section{J. Chabrowski (Brisbane) and P. Drábek (Plzen)}

\begin{abstract}
We study the existence of nonnegative solutions of elliptic equations involving concave and critical Sobolev nonlinearities. Applying various variational principles we obtain the existence of at least two nonnegative solutions.
\end{abstract}

1. Introduction. The main purpose of this paper is to investigate the existence of solutions of the following nonlinear elliptic problem:

$$
\left(1_{\varepsilon}\right) \quad\left\{\begin{array}{l}
-\Delta u+u=\varepsilon h(x) u^{q}+u^{2^{\star}-1} \quad \text { in } \mathbb{R}^{N}, \\
u>0, \quad h \in H^{1}\left(\mathbb{R}^{N}\right),
\end{array}\right.
$$

where $\varepsilon>0$ is a parameter, $0<q<1$ and $2^{\star}=2 N /(N-2), N \geq 3$, is the critical Sobolev exponent. We assume that $h$ is a nonnegative and $\not \equiv 0$ function in $L^{r}\left(\mathbb{R}^{N}\right) \cap C\left(\mathbb{R}^{N}\right)$, where $r=2^{\star} /\left(2^{\star}-q-1\right)$.

It is well known that equation $\left(1_{\varepsilon}\right)$ with $h \equiv 0$, that is,

$$
-\Delta u+u=u^{2^{\star}-1} \quad \text { in } \mathbb{R}^{N},
$$

does not have a positive solution. This is a consequence of the Pokhozhaev identity. By contrast, the equation

$$
-\Delta u=u^{2^{\star}-1} \quad \text { in } \mathbb{R}^{N}
$$

has a family of positive solutions $\varepsilon^{-(N-2) / 2} U((x-y) / \varepsilon)$, where the function $U$, called an instanton, is given by (see [17])

$$
U(x)=\left[\frac{N(N-2)}{N(N-2)+|x|^{2}}\right]^{(N-2) / 2} .
$$

We also have $\int_{\mathbb{R}^{N}}|\nabla U|^{2} d x=\int_{\mathbb{R}^{N}} U^{2^{\star}} d x=S^{N / 2}$. The constant $S$ is the best

2000 Mathematics Subject Classification: 35J20, 35J60, 35J70.

Key words and phrases: elliptic equations, critical Sobolev exponent, mountain-pass principle, local minimization, concentration-compactness principle.

Research of J. Chabrowski supported by Small ARC Grant.

Research of P. Drábek supported by the Grant Agency of the Czech Republic, Grant no. 201/00/0376. 
Sobolev constant given by

$$
S=\inf \left\{\int_{\mathbb{R}^{N}}|\nabla u|^{2} d x: u \in \mathcal{D}^{1,2}\left(\mathbb{R}^{N}\right), \int_{\mathbb{R}^{N}}|u|^{2^{\star}} d x=1\right\},
$$

where $\mathcal{D}^{1,2}\left(\mathbb{R}^{N}\right)$ denotes the Sobolev space obtained as the completion of the space $C_{0}^{1}\left(\mathbb{R}^{N}\right)$ with respect to the norm

$$
\|u\|_{\mathcal{D}^{1,2}}^{2}=\int_{\mathbb{R}^{N}}|\nabla u|^{2} d x
$$

However, the equation

$$
-\Delta u+a(x) u=u^{2^{\star}-1} \quad \text { in } \mathbb{R}^{N}
$$

with nonnegative and nonconstant coefficient $a(x)$ may have a positive solution. In fact, Benci-Cerami [6] proved the existence of a positive solution of (3) provided $\|a\|_{N / 2} \leq S\left(2^{N / 2}-1\right)$. This result has been extended in [9], where the multiplicity of solutions was expressed in terms of the category of the set $a^{-1}(0)$. Further results, under some integrability assumptions on the coefficient $a(x)$, can be found in [1], [13] and [14]. In Section 3 we show that problem $\left(1_{\varepsilon}\right)$, which is a small concave perturbation of $(1)$, admits a positive solution. This solution is obtained as a local minimizer of a variational functional for $\left(1_{\varepsilon}\right)$. In Section 4 , we consider an equation of the form

$$
\left(1_{\varepsilon, s}\right) \quad-\Delta u+\varepsilon^{s} u=\varepsilon h(x) u^{q}+u^{2^{\star}-1} \quad \text { in } \mathbb{R}^{N},
$$

where $s>1$. Theorem 4.1 of Section 4 gives the existence of two positive solutions: the first is a mountain-pass type solution and the second is obtained through local minimization of the variational functional for $\left(1_{\varepsilon, s}\right)$. In Section 5 we establish the existence of infinitely many solutions of equations $\left(1_{\varepsilon}\right)$ and $\left(1_{\varepsilon, s}\right)$ for $\varepsilon>0$ small. The results of Section 3 show that at least one of the solutions of the equation in $\left(1_{\varepsilon}\right)$ is positive. By Theorem 4.1 of Section 4 at least two solutions of $\left(1_{\varepsilon, s}\right)$ are positive under an additional assumption that $s<2 /(1-q)$. In Section 6 , we extend the local minimization to some nonlinear problems involving the $p$-Laplacian. Theorems 7.1 and 8.1 of Sections 7 and 8 , respectively, complement the results obtained in [16]. In the case of the $p$-Laplacian the exponent $q$ satisfies the inequality $q<p$ and the corresponding nonlinearity is not necessarily concave.

Throughout our paper we use standard notation and terminology. In a given Banach space $X$, we denote by "- w" weak convergence and by " $\rightarrow$ " strong convergence. For $u \in \mathbb{R}$, we let $u^{+}=\max (0, u)$ and $u^{-}=\max (0,-u)$.

Let $F \in C^{1}(X, \mathbb{R})$. A sequence $\left\{u_{m}\right\}$ is said to be a Palais-Smale sequence for $F$ at level $c\left((\mathrm{PS})_{c}\right.$ sequence for short) if $F\left(u_{m}\right) \rightarrow c$ and $F^{\prime}\left(u_{m}\right) \rightarrow 0$ in $X^{*}$ as $m \rightarrow \infty$. We say that $F$ satisfies the Palais-Smale condition at level $c\left((\mathrm{PS})_{c}\right.$ condition for short) if any $(\mathrm{PS})_{c}$ sequence is relatively compact in $X$. By $H^{1}\left(\mathbb{R}^{N}\right)$ we denote the usual Sobolev space 
equipped with the norm

$$
\|u\|^{2}=\int_{\mathbb{R}^{N}}\left(|\nabla u|^{2}+u^{2}\right) d x .
$$

2. Palais-Smale condition. For $u \in H^{1}\left(\mathbb{R}^{N}\right)$ we define the energy functional associated with $\left(1_{\varepsilon}\right)$ :

$$
J_{\varepsilon}(u)=\frac{1}{2} \int_{\mathbb{R}^{N}}\left(|\nabla u|^{2}+u^{2}\right) d x-\frac{\varepsilon}{q+1} \int_{\mathbb{R}^{N}} h(x)|u|^{q+1} d x-\frac{1}{2^{\star}} \int_{\mathbb{R}^{N}}|u|^{2^{\star}} d x .
$$

An elementary analysis of the real-valued function

$$
a \mapsto \frac{a^{2^{\star}}}{N}-\varepsilon\left(\frac{1}{q+1}-\frac{1}{2}\right)\|h\|_{r} a^{q+1}, \quad a \geq 0,
$$

shows that it has a global minimum greater than or equal to $-C^{*} \varepsilon^{r}$ with some $C^{*}=C^{*}\left(N, q,\|h\|_{r}\right)>0$. Thus we have

$$
\frac{a^{2^{\star}}}{N}-\varepsilon\left(\frac{1}{q+1}-\frac{1}{2}\right)\|h\|_{r} a^{q+1} \geq-C^{*} \varepsilon^{r}
$$

for every $a \geq 0$.

Proposition 2.1. The functional $J_{\varepsilon}$ satisfies the $(\mathrm{PS})_{c}$ condition for

$$
c<\frac{S^{N / 2}}{N}-\varepsilon^{r} C^{*} .
$$

Proof. If $\left\{u_{m}\right\}$ is a $(\mathrm{PS})_{c}$ sequence with $c$ satisfying (5), then $\left\{u_{m}\right\}$ is bounded in $H^{1}\left(\mathbb{R}^{N}\right)$. Indeed, there exists an integer $m_{0}$ such that

$$
\begin{aligned}
c+1+o\left(\left\|u_{m}\right\|\right) & \geq J_{\varepsilon}\left(u_{m}\right)-\frac{1}{2}\left\langle J_{\varepsilon}^{\prime}\left(u_{m}\right), u_{m}\right\rangle \\
& =\frac{q-1}{2(q+1)} \varepsilon \int_{\mathbb{R}^{N}} h\left|u_{n}\right|^{q+1} d x+\frac{1}{N} \int_{\mathbb{R}^{N}}\left|u_{m}\right|^{2^{\star}} d x
\end{aligned}
$$

for all $m \geq m_{0}$. By the Hölder and Sobolev inequalities, we deduce

$$
\int_{\mathbb{R}^{N}}\left|u_{m}\right|^{2^{\star}} d x \leq C_{1}+C_{2}\left\|u_{m}\right\|^{q+1}+o\left(\left\|u_{m}\right\|\right)
$$

for some constants $C_{1}, C_{2}>0$ and all $m \geq m_{0}$. On the other hand,

$$
\begin{aligned}
\frac{1}{2} \int_{\mathbb{R}^{N}}\left(\left|\nabla u_{m}\right|^{2}+u_{m}^{2}\right) d x & =J_{\varepsilon}\left(u_{m}\right)+\frac{\varepsilon}{q+1} \int_{\mathbb{R}^{N}} h\left|u_{m}\right|^{q+1} d x+\frac{1}{2^{\star}} \int_{\mathbb{R}^{N}}\left|u_{m}\right|^{2^{\star}} d x \\
& \leq c+1+\frac{\varepsilon}{q+1} \int_{\mathbb{R}^{N}} h\left|u_{m}\right|^{q+1} d x+\frac{1}{2^{\star}} \int_{\mathbb{R}^{N}}\left|u_{m}\right|^{2^{\star}} d x
\end{aligned}
$$

for $m \geq m_{0}$. Combining the last two estimates we easily derive the boundedness of $\left\{u_{m}\right\}$ in $H^{1}\left(\mathbb{R}^{N}\right)$. Therefore, we may assume that $u_{m} \rightarrow u$ in $H^{1}\left(\mathbb{R}^{N}\right), u_{m} \rightarrow u$ in $L^{p}(\Omega)$ for each $1 \leq p<2^{\star}$ and each bounded domain 
$\Omega \subset \mathbb{R}^{N}$. Also, we assume that $u_{m} \geq 0$ on $\mathbb{R}^{N}$. Otherwise we replace $J_{\varepsilon}$ by a functional

$$
J_{\varepsilon}^{+}(u)=\frac{1}{2} \int_{\mathbb{R}^{N}}\left(|\nabla u|^{2}+u^{2}\right) d x-\frac{\varepsilon}{q+1} \int_{\mathbb{R}^{N}} h\left(u^{+}\right)^{q+1} d x-\frac{1}{2^{\star}} \int_{\mathbb{R}^{N}}\left(u^{+}\right)^{2^{\star}} d x
$$

and observe that every bounded (PS $)_{c}$ sequence $\left\{u_{n}\right\}$ for $J_{\varepsilon}^{+}$has the property that $u_{n}^{-} \rightarrow 0$ in $H^{1}\left(\mathbb{R}^{N}\right)$. We set

$$
\begin{aligned}
& \mu_{\infty}=\lim _{R \rightarrow \infty} \limsup _{m \rightarrow \infty} \int_{|x| \geq R}\left(\left|\nabla u_{m}\right|^{2}+u_{m}^{2}\right) d x, \\
& \nu_{\infty}=\lim _{R \rightarrow \infty} \limsup _{m \rightarrow \infty} \int_{|x| \geq R}\left|u_{m}\right|^{2^{\star}} d x .
\end{aligned}
$$

Then by the concentration-compactness principle (see [15] and [8]) we have

$$
\begin{gathered}
\left|\nabla u_{m}\right|^{2}+u_{m}^{2} \rightarrow d \mu=|\nabla u|^{2}+u^{2}+\sum_{j \in J} \mu_{j} \delta_{x_{j}}+\mu_{\infty} \delta_{\infty}, \\
\left|u_{m}\right|^{2^{\star}} \rightarrow d \nu=|u|^{2^{\star}}+\sum_{j \in J} \nu_{j} \delta_{x_{j}}+\nu_{\infty} \delta_{\infty},
\end{gathered}
$$

where $J$ is at most a countable set, and $\nu_{j}, \mu_{j}>0$ are constants satisfying

$$
S \nu_{j}^{(N-2) / N} \leq \mu_{j}
$$

for $j \in J \cup\{\infty\}$. Using a family of test functions concentrating at $x_{j}$ and a family of test functions concentrating at $\infty$, we check as in [2] and [8] that

$$
\nu_{j}=\mu_{j}
$$

for all $j \in J \cup\{\infty\}$. We now observe that if $\nu_{j} \neq 0$ for some $j \in J \cup\{\infty\}$, then by (6) and (7) we have

$$
\nu_{j} \geq S^{N / 2} .
$$

We show that $\nu_{j}=0$ for every $j \in J \cup\{\infty\}$. Arguing by contradiction, assume that $\nu_{j}>0$ for some $j \in J \cup\{\infty\}$. Then we have

$$
J_{\varepsilon}\left(u_{m}\right)-\frac{1}{2}\left\langle J_{\varepsilon}^{\prime}\left(u_{m}\right), u_{m}\right\rangle=\frac{1}{N} \int_{\mathbb{R}^{N}}\left|u_{m}\right|^{2^{\star}} d x+\frac{(q-1) \varepsilon}{2(q+1)} \int_{\mathbb{R}^{N}} h(x)\left|u_{m}\right|^{q+1} d x .
$$

Letting $m \rightarrow \infty$ and using (8) we deduce that

$$
\begin{aligned}
c & =\lim _{m \rightarrow \infty}\left(J_{\varepsilon}\left(u_{m}\right)-\frac{1}{2}\left\langle J_{\varepsilon}^{\prime}\left(u_{m}\right), u_{m}\right\rangle\right) \\
& \geq \frac{1}{N} \int_{\mathbb{R}^{N}}|u|^{2^{\star}} d x+\frac{S^{N / 2}}{N}+\frac{(q-1) \varepsilon}{2(q+1)} \int_{\mathbb{R}^{N}} h(x)|u|^{q+1} .
\end{aligned}
$$


If $u \equiv 0$, we deduce from (9) that $c \geq S^{N / 2} / N$, which is impossible. If $u \not \equiv 0$, we then derive from (9) and (4) that

$$
c \geq \frac{S^{N / 2}}{N}-C^{*} \varepsilon^{r},
$$

which is impossible. Since $\nu_{j}=0$ for every $j \in J \cup\{\infty\}$, we see that $u_{m} \rightarrow u$ in $L^{2^{\star}}\left(\mathbb{R}^{N}\right)$. To show that $u_{m} \rightarrow u$ in $H^{1}\left(\mathbb{R}^{N}\right)$ we write

$$
\begin{aligned}
o(1)= & \left\langle J_{\varepsilon}^{\prime}\left(u_{n}\right)-J_{\varepsilon}^{\prime}\left(u_{m}\right), u_{n}-u_{m}\right\rangle \\
= & \int_{\mathbb{R}^{N}}\left(\left|\nabla\left(u_{n}-u_{m}\right)\right|^{2}+\left(u_{n}-u_{m}\right)^{2}\right) d x \\
& -\varepsilon \int_{\mathbb{R}^{N}} h\left(\left|u_{n}\right|^{q-1} u_{n}-\left|u_{m}\right|^{q-1} u_{m}\right)\left(u_{n}-u_{m}\right) d x \\
& -\int_{\mathbb{R}^{N}}\left(\left|u_{n}\right|^{2^{\star}-2} u_{n}-\left|u_{m}\right|^{2^{\star}-2} u_{m}\right)\left(u_{n}-u_{m}\right) d x .
\end{aligned}
$$

Since $u_{n} \rightarrow u$ in $L^{2^{\star}}\left(\mathbb{R}^{N}\right)$, it is easy to check that the last two integrals converge to 0 as $n, m \rightarrow \infty$. Hence $\left\{u_{n}\right\}$ satisfies the Cauchy condition in $H^{1}\left(\mathbb{R}^{N}\right)$ and the convergence of $\left\{u_{n}\right\}$ in $H^{1}\left(\mathbb{R}^{N}\right)$ follows.

3. Local minimum. We are now in a position to establish the existence of a local minimum for $J_{\varepsilon}$.

THEOREM 3.1. There exists an $\varepsilon_{0}>0$ such that for each $0<\varepsilon \leq \varepsilon_{0}$ problem $\left(1_{\varepsilon}\right)$ has a solution $u_{\varepsilon}$ which is a local minimum of $J_{\varepsilon}$.

Proof. Using the Sobolev embedding theorem and the Hölder inequality we obtain the following estimate from below for the functional $J_{\varepsilon}$ :

$$
\begin{aligned}
J_{\varepsilon}(u) & \geq \frac{1}{2}\|u\|^{2}-\frac{\varepsilon}{q+1}\|h\|_{r}\|u\|^{q+1}-\frac{S^{-2^{\star} / 2}}{2^{\star}}\|u\|^{2^{\star}} \\
& =\|u\|^{2}\left(\frac{1}{2}-\frac{\varepsilon}{q+1}\|h\|_{r}\|u\|^{q-1}-\frac{S^{-2^{\star} / 2}}{2^{\star}}\|u\|^{2^{\star}-1}\right) .
\end{aligned}
$$

Let $\|u\|=\varrho$ and choose $\varrho>0$ so that

$$
\frac{1}{2}-\frac{S^{-2^{\star} / 2}}{2^{\star}} \varrho^{2^{\star}-1}>\varrho_{0}>0,
$$

where $\varrho_{0}$ is a constant. It then follows from the last two estimates that there exist constants $\varepsilon_{0}>0$ and $c_{1}>0$ such that

$$
J_{\varepsilon}(u) \geq c_{1} \varrho^{2}
$$

for all $\|u\|=\varrho$ and $0<\varepsilon \leq \varepsilon_{0}$. If $\varphi>0, \varphi \in H^{1}\left(\mathbb{R}^{N}\right)$, then

$$
J_{\varepsilon}(t \varphi)=\frac{t^{2}}{2} \int_{\mathbb{R}^{N}}\left(|\nabla \varphi|^{2}+\varphi^{2}\right) d x-\frac{t^{q+1} \varepsilon}{q+1} \int_{\mathbb{R}^{N}} h(x) \varphi^{q+1} d x-\frac{t^{2^{\star}}}{2^{\star}} \int_{\mathbb{R}^{N}} \varphi^{2^{\star}} d x<0
$$


for $t>0$ sufficiently small. Therefore, we have

$$
M=\inf _{\|u\| \leq \varrho} J_{\varepsilon}(u)<0 .
$$

We can also assume that $\varepsilon_{0}$ is chosen so that $S^{N / 2} / N-\varepsilon^{r} C^{*} \geq 0$ for $0<\varepsilon \leq \varepsilon_{0}$. By the Ekeland variational principle [11] there exists a minimizing sequence $\left\{u_{m}\right\} \subset B(0, \varrho)$ such that $J_{\varepsilon}\left(u_{m}\right) \rightarrow M$ and $J_{\varepsilon}^{\prime}\left(u_{m}\right) \rightarrow 0$ in $H^{-1}\left(\mathbb{R}^{N}\right)$. Since the functional $J_{\varepsilon}$ satisfies the $(\mathrm{PS})_{M}$ condition it is clear from the above construction that the functional $J_{\varepsilon}$ achieves a minimum $u_{\varepsilon}$ at an interior point of $B(0, \varrho)$. Since $J_{\varepsilon}\left(u_{\varepsilon}\right)=J_{\varepsilon}\left(\left|u_{\varepsilon}\right|\right)$ we may assume that $u_{\varepsilon} \geq 0$ and by the maximum principle we have $u_{\varepsilon}>0$ on $\mathbb{R}^{N}$. This completes the proof.

REMARK 3.2. It follows from the Hölder and Sobolev inequalities that $\left\|u_{\varepsilon}\right\| \leq r(\varepsilon)$ with $r(\varepsilon) \rightarrow 0$ as $\varepsilon \rightarrow 0$.

Indeed, since $u_{\varepsilon}$ satisfies $\left(1_{\varepsilon}\right)$ we deduce from the inequality

$$
J_{\varepsilon}\left(u_{\varepsilon}\right)-\frac{1}{2}\left\langle J_{\varepsilon}^{\prime}\left(u_{\varepsilon}\right), u_{\varepsilon}\right\rangle<0
$$

that

$$
\int_{\mathbb{R}^{N}} u_{\varepsilon}^{2^{\star}} d x \leq N \varepsilon \frac{1-q}{2(q+1)} \int_{\mathbb{R}^{N}} h u_{\varepsilon}^{q+1} d x \leq N \varepsilon \frac{1-q}{2(q+1)}\|h\|_{r}\left(\int_{\mathbb{R}^{N}} u_{\varepsilon}^{2^{\star}} d x\right)^{(q+1) / 2^{\star}}
$$

and hence

$$
\left(\int_{\mathbb{R}^{N}} u_{\varepsilon}^{2^{\star}} d x\right)^{\left(2^{\star}-q-1\right) / 2^{\star}} \leq N \varepsilon \frac{1-q}{2(q+1)}\|h\|_{r} .
$$

Combining this with the inequality $J_{\varepsilon}\left(u_{\varepsilon}\right)<0$ we derive our assertion.

REMARK 3.3. Theorem 3.1 remains true if $h$ changes sign, that is, $h^{+} \not \equiv 0$ and $h^{-} \not \equiv 0$.

This follows from the fact that positivity of $h$ was only used to show that $\inf _{\|u\| \leq \varrho} J_{\varepsilon}(u)<0$ for small $\varrho>0$. This also can be shown by choosing $\varphi \in H^{1}\left(\mathbb{R}^{N}\right), \varphi \geq 0, \not \equiv 0$ and with $\operatorname{supp} \varphi \subset\{x: h(x)>0\}$. This obviously implies that $J_{\varepsilon}(t \varphi)<0$ for small $t>0$.

In Proposition 3.4 below, we show that problem $\left(1_{\varepsilon}\right)$ has no solution for $\varepsilon$ large.

Proposition 3.4. There exists $\varepsilon^{*}>0$ such that problem $\left(1_{\varepsilon}\right)$ has no solution for $\varepsilon \geq \varepsilon^{*}$.

Proof. We follow the argument from [12]. Suppose that problem $\left(1_{\varepsilon}\right)$ has a solution $u_{\varepsilon}$ for every $\varepsilon>0$. Let $\varepsilon_{k} \rightarrow \infty$ and set $u_{k}=u_{\varepsilon_{k}}$. Since $h \geq 0$ and $\not \equiv 0$ on $\mathbb{R}^{N}$, we may assume without loss of generality that $h(x)>0$ on $\overline{B(0, R)}$ for some $R>0$. We denote by $\lambda_{1}(R)>0$ the first eigenvalue of the 
problem

$$
-\Delta u+u=\lambda u, \quad u \in W_{0}^{1,2}(B(0, R)) .
$$

Given $\delta>0$ we choose $\varepsilon_{k}$ sufficiently large so that

$$
\varepsilon_{k} h(x) u^{q}+u^{2^{\star}} \geq\left(\lambda_{1}(R)+\delta\right) u
$$

for all $u \geq 0$ and $x \in \overline{B(0, R)}$. Then $u_{k}$ is a supersolution of the problem

$$
-\Delta u+u=\left(\lambda_{1}(R)+\delta\right) u, \quad u \in W_{0}^{1,2}(B(0, R)) .
$$

If $\varphi>0$ is an eigenfunction corresponding to $\lambda_{1}(R)$, then for each $t>0, t \varphi$ is a subsolution of (11). We now choose $t>0$ sufficiently small so that $t \varphi \leq u_{k}$ on $\overline{B(0, R)}$. Hence we can find a solution $v$ of (11) such that $t \varphi \leq v \leq u_{k}$ on $B(0, R)$. However, this is impossible for $\delta>0$ sufficiently small, since the first eigenvalue of (10) is isolated.

4. Mountain-pass solution. Inspection of the proofs of Proposition 2.1 and Theorem 3.1 shows that the method of local minimization can be extended to problem $\left(1_{\varepsilon, s}\right)$. A variational functional for problem $\left(1_{\varepsilon, s}\right)$ has the form

$$
J_{\varepsilon, s}(u)=\frac{1}{2} \int_{\mathbb{R}^{N}}\left(|\nabla u|^{2}+\varepsilon^{s} u^{2}\right) d x-\frac{\varepsilon}{q+1} \int_{\mathbb{R}^{N}} h(x)|u|^{q+1} d x-\frac{1}{2^{\star}} \int_{\mathbb{R}^{N}}|u|^{2^{\star}} d x .
$$

Using the mountain-pass principle [3] and local minimization we show that problem $\left(1_{\varepsilon, s}\right)$ has two distinct positive solutions.

TheOREM 4.1. Suppose that $N \geq 5$ and $1<s<2 /(1-q)$. Then there exists $\bar{\varepsilon}>0$ such that for every $0<\varepsilon \leq \bar{\varepsilon}$ problem $\left(1_{\varepsilon, s}\right)$ admits two distinct positive solutions.

Proof. First, we check that the functional $J_{\varepsilon, s}$ has mountain-pass geometry. We set

$$
\|u\|_{\varepsilon}^{2}=\int_{\mathbb{R}^{N}}\left(|\nabla u|^{2}+\varepsilon^{s} u^{2}\right) d x
$$

for $\varepsilon>0$. This is a norm equivalent to $\|\cdot\|$. First, we notice that if $\|v\|=\varrho$, then $\left\|v^{\varepsilon}\right\|_{\varepsilon}=\varrho \varepsilon^{s / 2}$, where $v^{\varepsilon}(x)=\varepsilon^{N s / 4} v\left(\varepsilon^{s / 2} x\right)$. We now consider a sphere $\|u\|_{\varepsilon}=\varrho \varepsilon^{s / 2}$ in $H^{1}\left(\mathbb{R}^{N}\right)$, where $\varrho>0$ is fixed. For $\|u\|_{\varepsilon}=\varrho \varepsilon^{s / 2}$ we have the following estimate:

$$
\begin{aligned}
J_{\varepsilon, s}(u) & \geq \varepsilon^{s} \varrho^{2} / 2-C_{1} \varepsilon^{1+(q+1) s / 2} \varrho^{q+1}-C_{2} \varepsilon^{s 2^{\star} / 2} \varrho^{2^{\star}} \\
& =\varepsilon^{s}\left(\varrho^{2} / 2-C_{1} \varepsilon^{1+(q-1) s / 2} \varrho^{q+1}-C_{2} \varepsilon^{s\left(2^{\star}-2\right) / 2} \varrho^{2^{\star}}\right) .
\end{aligned}
$$

From this and $s<2 /(1-q)$ we deduce that there exists $\varepsilon_{1}>0$ such that to every $0<\varepsilon \leq \varepsilon_{1}$ there corresponds $\varrho_{\varepsilon}>0$ such that $J_{\varepsilon, s}(u) \geq \varrho_{\varepsilon}$ for $\|u\|_{\varepsilon}=\varrho \varepsilon^{s / 2}$. It is clear that $J_{\varepsilon, s}(t U)<0$ for $t>0$ sufficiently large, where $U$ is the instanton defined in Section 1. We point out here that $J_{\varepsilon, s}(U)$ is 
well defined since $N \geq 5$. Therefore, we can define the mountain-pass level

$$
c_{\varepsilon}=\inf _{\gamma \in \Gamma} \max _{0 \leq t \leq 1} J_{\varepsilon, s}(u),
$$

where

$$
\Gamma=\left\{\gamma \in C\left([0,1], H^{1}\left(\mathbb{R}^{N}\right)\right): \gamma(0)=0, \gamma(1)<0\right\} .
$$

We now show that

$$
c_{\varepsilon}<\frac{S^{N / 2}}{N}-C^{*} \varepsilon^{r}
$$

for $\varepsilon>0$ sufficiently small. First, we choose $t^{*}>0$ and $\varepsilon^{*}>0$ small enough so that

$$
J_{\varepsilon, s}(t U) \leq \frac{S^{N / 2}}{N}-C^{*} \varepsilon^{r}
$$

for $0 \leq t \leq t^{*}$ and $0<\varepsilon \leq \varepsilon^{*}$. Here $\varepsilon^{*}$ is chosen so that

$$
\frac{S^{N / 2}}{N}-C^{*} \varepsilon^{r} \geq c_{0}>0
$$

for all $0<\varepsilon \leq \varepsilon^{*}$. To estimate $J_{\varepsilon, s}(t U)$ for $t^{*} \leq t$ we observe that the function

$$
t \mapsto \frac{t^{2}}{2} \int_{\mathbb{R}^{N}}\left(|\nabla U|^{2}+\varepsilon^{s} U^{2}\right) d x-\frac{t^{2^{\star}}}{2^{\star}} \int_{\mathbb{R}^{N}} U^{2^{\star}} d x, \quad t \geq 0
$$

achieves its maximum on $[0, \infty)$ at a point $t_{\varepsilon}>0$. If $\varepsilon=0$, then $t_{0}=1$ and we also have $1 \leq t_{\varepsilon} \leq t_{1}$ for $0 \leq \varepsilon \leq 1$. We then have, for $t^{*} \leq t$,

$$
\begin{aligned}
J_{\varepsilon, s}(t U) \leq & \frac{t_{\varepsilon}^{2}}{2} \int_{\mathbb{R}^{N}}\left(|\nabla U|^{2}+\varepsilon^{s} U^{2}\right) d x-\frac{t_{\varepsilon}^{2^{\star}}}{2^{\star}} \int_{\mathbb{R}^{N}} U^{2^{\star}} d x \\
& -\frac{\varepsilon\left(t^{*}\right)^{q+1}}{q+1} \int_{\mathbb{R}^{N}} h(x) U^{q+1} d x .
\end{aligned}
$$

Since

$$
\frac{t_{\varepsilon}^{2}}{2} \int_{\mathbb{R}^{N}}|\nabla U|^{2} d x-\frac{t_{\varepsilon}^{2^{\star}}}{2^{\star}} \int_{\mathbb{R}^{N}} U^{2^{\star}} d x \leq \frac{1}{2} \int_{\mathbb{R}^{N}}|\nabla U|^{2} d x-\frac{1}{2^{\star}} \int_{\mathbb{R}^{N}} U^{2^{\star}} d x=\frac{S^{N / 2}}{N}
$$

we deduce from the previous inequality that

$$
J_{\varepsilon, s}(t U) \leq \frac{S^{N / 2}}{N}+O\left(\varepsilon^{s}\right)-\frac{\varepsilon\left(t^{*}\right)^{q+1}}{q+1} \int_{\mathbb{R}^{N}} h(x) U^{q+1} d x
$$

for $t^{*} \leq t$ and $0<\varepsilon \leq \varepsilon^{*}$. Taking $\varepsilon^{*}$ smaller if necessary, we can assume

$$
O\left(\varepsilon^{s}\right)-\frac{\varepsilon\left(t^{*}\right)^{q+1}}{q+1} \int_{\mathbb{R}^{N}} h(x) U^{q+1} d x \leq-C^{*} \varepsilon^{r}
$$

for all $0<\varepsilon \leq \varepsilon^{*}$. Combining (13)-(15) we obtain (12). The argument used in the proof of Proposition 2.1 shows that the functional $J_{\varepsilon, s}$ satisfies 
the $(\mathrm{PS})_{c}$ condition with $c$ satisfying (5). This completes the proof of the existence of a mountain-pass solution. The above estimate of the functional $J_{\varepsilon, s}$ on the sphere $\|u\|_{\varepsilon}=\varrho \varepsilon^{s / 2}$ shows that the second solution can be obtained by local minimization as in Theorem 3.1.

In the next result we examine the behaviour, as $\varepsilon \rightarrow 0$, of the solutions from Theorem 4.1.

Proposition 4.2. Suppose that the assumptions of Theorem 4.1 hold.

(i) If $\left\{u_{\varepsilon}\right\}, 0<\varepsilon \leq \bar{\varepsilon}$, are mountain-pass solutions of problem $\left(1_{\varepsilon, s}\right)$, then $\lim _{\varepsilon \rightarrow 0} \varepsilon^{-s}\left\|u_{\varepsilon}\right\|^{2}=\infty$.

(ii) If $\left\{u_{\varepsilon}\right\}, 0<\varepsilon \leq \bar{\varepsilon}$, are solutions of problem $\left(1_{\varepsilon, s}\right)$ which are local minimizers, then $\lim _{\varepsilon \rightarrow 0}\left\|u_{\varepsilon}\right\|=0$.

Proof. It follows from the proof of Theorem 4.1 that for every $\varrho>0$ there exists an $\varepsilon_{0}>0$ such that a mountain-pass solution $u_{\varepsilon}$ satisfies

$$
J_{\varepsilon, s}\left(u_{\varepsilon}\right) \geq \frac{\varepsilon^{s} \varrho^{2}}{4} \quad \text { for } 0<\varepsilon \leq \varepsilon_{0} .
$$

This implies that

$$
\begin{aligned}
\frac{1}{2} \int_{\mathbb{R}^{N}}\left(\left|\nabla u_{\varepsilon}\right|^{2}+\varepsilon^{s} u_{\varepsilon}^{2}\right) d x & \geq \frac{\varepsilon^{s} \varrho^{2}}{4}+\frac{\varepsilon}{2^{\star}} \int_{\mathbb{R}^{N}} h u_{\varepsilon}^{q+1} d x+\frac{1}{2^{\star}} \int_{\mathbb{R}^{N}} u_{\varepsilon}^{2^{\star}} d x \\
& =\frac{\varepsilon^{s} \varrho^{2}}{4}+\frac{1}{2^{\star}} \int_{\mathbb{R}^{N}}\left(\left|\nabla u_{\varepsilon}\right|^{2}+\varepsilon^{s} u_{\varepsilon}^{2}\right) d x .
\end{aligned}
$$

From this we deduce that

$$
\frac{1}{N} \int_{\mathbb{R}^{N}}\left(\left|\nabla u_{\varepsilon}\right|^{2}+\varepsilon^{s} u_{\varepsilon}^{2}\right) d x \geq \frac{\varepsilon^{s} \varrho^{2}}{4}
$$

and the result follows.

(ii) Following the argument of Remark 3.2 we see that

$$
J_{\varepsilon, s}\left(u_{\varepsilon}\right)-\frac{1}{2}\left\langle J_{\varepsilon, s}^{\prime}\left(u_{\varepsilon}\right), u_{\varepsilon}\right\rangle<0
$$

implies that

$$
\int_{\mathbb{R}^{N}} u_{\varepsilon}^{2^{\star}} d x \leq N \frac{1-q}{2(q+1)} \varepsilon\|h\|_{r}\left\|u_{\varepsilon}\right\|_{\varepsilon}^{q+1} .
$$

We then have

$$
\begin{aligned}
\frac{1}{2} \int_{\mathbb{R}^{N}}\left(\left|\nabla u_{\varepsilon}\right|^{2}+\varepsilon^{s} u_{\varepsilon}^{2}\right) d x & =J_{\varepsilon, s}\left(u_{\varepsilon}\right)+\frac{\varepsilon}{q+1} \int_{\mathbb{R}^{N}} h u_{\varepsilon}^{q+1} d x+\frac{1}{2^{\star}} \int_{\mathbb{R}^{N}} u_{\varepsilon}^{2^{\star}} d x \\
& \leq \frac{\varepsilon}{q+1}\|h\|_{r}\left\|u_{\varepsilon}\right\|_{\varepsilon}^{q+1}+\frac{1}{2^{\star}} \int_{\mathbb{R}^{N}} u_{\varepsilon}^{2^{\star}} d x \leq \varepsilon C_{1}\left\|u_{\varepsilon}\right\|_{\varepsilon}^{q+1}
\end{aligned}
$$


for some constant $C_{1}>0$ independent of $\varepsilon$. Since

$$
\varepsilon^{s / 2}\|u\| \leq\|u\|_{\varepsilon} \leq\|u\|
$$

for every $u \in H^{1}\left(\mathbb{R}^{N}\right)$, we deduce from the last estimate for $u_{\varepsilon}$ that

$$
\left\|u_{\varepsilon}\right\| \leq C \varepsilon^{1 /(1-q)-s / 2}
$$

for some constant $C>0$ independent of $\varepsilon$. This estimate completes the proof of assertion (ii).

REMARK 4.3. Theorem 4.1 continues to hold if $h$ changes sign and satisfies $\int_{\mathbb{R}^{N}} h(x) U^{q+1} d x>0$.

This assumption allows one to show that the mountain-pass level $c_{\varepsilon}$ for the functional $J_{\varepsilon, s}$ satisfies (12).

5. Existence of infinitely many solutions. Since the right-hand side of the equation in $\left(1_{\varepsilon}\right)$ involves concave and convex nonlinearities we can establish the existence of infinitely many solutions. Our approach is based on the Bartsch-Willem fountain theorem [4].

Let $\left\{e_{k}\right\}, k=1,2, \ldots$, be an orthonormal basis for $H^{1}\left(\mathbb{R}^{N}\right)$. We set

$$
X(j)=\operatorname{span}\left(e_{1}, \ldots, e_{j}\right), \quad X_{k}=\bigoplus_{j \geq k} X(j), \quad X^{k}=\bigoplus_{j \leq k} X(j)
$$

for each $k \in \mathbb{N}$.

Theorem 5.1 (Bartsch-Willem [5]). Let $F: H^{1}\left(\mathbb{R}^{N}\right) \rightarrow \mathbb{R}$ be a $C^{1}$ even functional satisfying the following conditions:

$\left(A_{1}\right) \quad$ There exists an integer $k_{0}$ such that for every $k \geq k_{0}$ there exists $R_{k}>0$ such that $F(u) \geq 0$ for every $u \in X_{k}$ with $\|u\|=R_{k}$.

$\left(A_{2}\right) \quad b_{k}=\inf _{B_{k}} F(u) \rightarrow 0$ as $k \rightarrow \infty$, where $B_{k}=\left\{u \in X_{k}:\|u\| \leq R_{k}\right\}$.

$\left(A_{3}\right) \quad$ For every $k \geq 1$ there exist $r_{k} \in\left(0, R_{k}\right)$ and $d_{k}<0$ such that $F(u) \leq d_{k}$ for every $u \in X^{k}$ with $\|u\|=r_{k}$.

$\left(A_{4}\right) \quad$ Every sequence $u_{n} \in X^{n}$ with $F\left(u_{n}\right)<0$ and $\left.F^{\prime}\right|_{X^{n}} \rightarrow 0$ as $n \rightarrow \infty$ has a subsequence which converges to a critical point of $F$.

Then for each $k \geq k_{0}, F$ has a critical value $c_{k} \in\left[b_{k}, d_{k}\right]$.

THEOREM 5.2. There exists $\varepsilon_{0}>0$ such that for $0<\varepsilon \leq \varepsilon_{0}$ the equation in $\left(1_{\varepsilon}\right)$ admits infinitely many solutions.

Proof. It suffices to check that the functional $J_{\varepsilon}$ satisfies the assumptions of Theorem 5.1. For each $k \in \mathbb{N}$ we define

$$
\lambda_{k}=\sup _{u \in X_{k}-\{0\}} \frac{\left(\int_{\mathbb{R}^{N}} h|u|^{q+1} d x\right)^{1 /(q+1)}}{\|u\|} .
$$


It is clear that $\left\{\lambda_{k}\right\}$ is a decreasing sequence. Since $u \mapsto \int_{\mathbb{R}^{N}} h(x)|u(x)|^{q+1} d x$ is a completely continuous functional on $H^{1}\left(\mathbb{R}^{N}\right)$, we can show as in [18] that $\lambda_{k} \rightarrow 0$ as $k \rightarrow \infty$. We now proceed as in the proof of Theorem 3 in [5]. Let $u \in H^{1}\left(\mathbb{R}^{N}\right)$. Then we have

$$
J_{\varepsilon}(u) \geq \frac{1}{2}\|u\|^{2}-\frac{\lambda_{k}^{q+1} \varepsilon}{q+1}\|u\|^{q+1}-\frac{S^{-2^{\star} / 2}}{2^{\star}}\|u\|^{2^{\star}} .
$$

If $\|u\|<R$ with $R>0$ small, then

$$
\frac{S^{-2^{\star} / 2}}{2^{\star}}\|u\|^{2^{\star}} \leq \frac{1}{4}\|u\|^{2} .
$$

We set $R_{k}=\left(4 \varepsilon \lambda_{k}^{q+1} /(q+1)\right)^{1 /(1-q)}$. We see that $R_{k}$ satisfies

$$
\frac{R_{k}^{2}}{4}=\frac{\varepsilon}{q+1} \lambda_{k}^{q+1} R_{k}^{q+1}
$$

and moreover $R_{k} \rightarrow 0$ as $k \rightarrow \infty$. Therefore we can find $k_{0} \in \mathbb{N}$ such that $R_{k} \leq R$ for $k \geq k_{0}$. Consequently, if $u \in X_{k}, k \geq k_{0}$, satisfies $\|u\|=R_{k}$, we then have

$$
J_{\varepsilon}(u) \geq \frac{1}{4}\|u\|^{2}-\frac{\varepsilon \lambda_{k}^{q+1}}{q+1}\|u\|^{q+1}=0 .
$$

This shows that $\left(A_{1}\right)$ holds and since $R_{k} \rightarrow 0$, condition $\left(A_{2}\right)$ is also satisfied. To check $\left(A_{3}\right)$ we observe that on the finite-dimensional space $X^{k}$ all norms are equivalent. Hence

$$
J_{\varepsilon}(u) \leq \frac{1}{2}\|u\|^{2}-A\|u\|^{q+1}-B\|u\|^{2^{\star}}
$$

for some constants $A, B>0$. Since $q+1<2$, taking $r_{k}$ sufficiently small, we can satisfy $\left(A_{3}\right)$. The Palais-Smale condition $\left(A_{4}\right)$ follows from Proposition 2.1. We only need to select $\varepsilon_{0}>0$ so that $S^{N / 2} / N-\varepsilon^{r} C^{*}>0$ for $0<\varepsilon \leq \varepsilon_{0}$.

A similar argument can be employed to show the existence of infinitely many solutions of equation $\left(1_{\varepsilon, s}\right)$.

TheOREM 5.3. Let $s>1$. There exists $\bar{\varepsilon}>0$ such that equation $\left(1_{\varepsilon, s}\right)$ for $0<\varepsilon \leq \bar{\varepsilon}$ has infinitely many solutions.

6. The $p$-Laplacian. In this section we study the problem

$$
\left(1_{\lambda}\right) \quad\left\{\begin{array}{l}
-\Delta_{p} u+u^{p-1}=u^{p^{\star}-1}+\lambda f(x, u) \text { in } \mathbb{R}^{N}, \\
u \geq 0 \text { on } \mathbb{R}^{N},
\end{array}\right.
$$

where $\Delta_{p}=-\operatorname{div}\left(|\nabla u|^{p-2} \nabla u\right)$ is the $p$-Laplacian of $u, p^{\star}=N p /(N-p)$, $N>p$, is the critical Sobolev exponent and $\lambda>0$ is a positive parameter.

We assume that the function $f: \mathbb{R}^{N} \times \mathbb{R} \rightarrow \mathbb{R}$ satisfies the following conditions: 
$\left(f_{1}\right) \quad f \in C\left(\mathbb{R}^{N} \times \mathbb{R}, \mathbb{R}\right)$ and $f(x, 0)=0$ for $x \in \mathbb{R}^{N}$,

$\left(f_{2}\right) \quad$ for every $R>0$ there exist $\theta_{R} \in\left[p, p^{\star}\right)$ and constants $a_{R}, b_{R}>0$ such that

$$
|f(x, s)| \leq a_{R} s^{\theta_{R}-1}+b_{R}
$$

for every $|x| \leq R$ and $s \geq 0$,

$\left(f_{3}\right) \quad$ there exist constants $r_{1} \in\left(1, p^{\star}\right)$ and $r_{2} \in\left(p, p^{\star}\right)$ such that

$$
f(x, s) \leq b(x) s^{r_{1}-1}+c s^{r_{2}-1}
$$

for every $x \in \mathbb{R}^{N}$ and $s \geq 0$, where $b \in L^{p^{\star} /\left(p^{\star}-r_{1}\right)}\left(\mathbb{R}^{N}\right)$ and $b(x) \geq 0$ on $\mathbb{R}^{N}$ and $c>0$ is a constant. Furthermore, we assume that there exist $q \in(1, p)$ and an open set $\Omega \subset \mathbb{R}^{N}$ such that the function $F(x, s)=\int_{0}^{s} f(x, t) d t$ satisfies

$$
F(x, s) \geq a s^{q}
$$

for every $x \in \Omega$ and $s \geq 0$ and some positive constant $a>0$.

An example of the nonlinearity $f$ satisfying the above conditions is

$$
f(x, s)=b(x) s^{q-1}-d(x) s^{l-1}+C s^{r_{2}-1},
$$

where $C>0$ is a constant, $1 \leq l<p^{\star}, b(x) \geq 0$ on $\mathbb{R}^{N}$ and belongs to $L^{p^{\star} /\left(p^{\star}-q\right)}\left(\mathbb{R}^{N}\right) \cap C\left(\mathbb{R}^{N}\right)$ and $d(x)$ is a continuous and nonnegative function on $\mathbb{R}^{N}$. Moreover, it is assumed that $b(x) \geq a_{0}>0$ on $B(0, R)$ and $d(x)=0$ on $B(0, R)$. In this example $q=r_{1}$ and $\Omega=B(0, R)$.

A similar problem

$$
\left\{\begin{array}{l}
-\Delta_{p} u=u^{p^{\star}-1}+\lambda f(x, u) \text { in } \mathbb{R}^{N}, \\
u \geq 0 \text { on } \mathbb{R}^{N}
\end{array}\right.
$$

has been studied in [16]. Under some additional assumptions, guaranteeing the mountain-pass geometry of the variational functional for this problem, the authors established the existence of a nontrivial solution. First, we establish the existence of a solution for problem $\left(1_{\lambda}\right)$ through local minimization. Under assumptions $\left(f_{1}\right),\left(f_{2}\right)$ and $\left(f_{3}\right)$ a variational functional corresponding to $\left(1_{\lambda}\right)$ is not well defined on $W^{1, p}\left(\mathbb{R}^{N}\right)$. Therefore, following the paper [16] we truncate the nonlinearity $f$. We also point out here that in [16] the constant $q$ (see assumption $\left(f_{3}\right)$ ) satisfies $1<q<p^{\star}$ and some other restrictions. This assumption is replaced here by $1<q<p$, since we construct a solution through local minimization (see Lemma 6.2 below).

Let $\varphi \in C^{1}\left(\mathbb{R}^{N}\right)$ be a function such that $\varphi(x)=1$ for $|x| \leq 1, \varphi(x)=0$ for $|x| \geq 2$ and $0 \leq \varphi(x) \leq 1$ on $\mathbb{R}^{N}$. We set $\varphi_{n}(x)=\varphi(x / n)$ and extend $f$ by 0 for $s \leq 0$, that is, $f(x, s)=0$ for $x \in \mathbb{R}^{N}$ and $s \leq 0$. We define a modified nonlinearity $f_{n}(x, s)=\varphi_{n}(x) f(x, s)$. For each $n \in \mathbb{N}$ we consider the following problem: 


$$
\left(1_{\lambda, n}\right) \quad\left\{\begin{array}{l}
-\Delta_{p} u+u^{p-1}=u^{p^{\star}-1}+\lambda f_{n}(x, u) \text { in } \mathbb{R}^{N}, \\
u \geq 0 \text { on } \mathbb{R}^{N} .
\end{array}\right.
$$

Let $F_{n}(x, s)=\int_{0}^{s} f_{n}(x, t) d t$. We associate with problem $\left(1_{\lambda, n}\right)$ the variational functional

$$
I_{\lambda, n}(u)=\frac{1}{p} \int_{\mathbb{R}^{N}}\left(|\nabla u|^{p}+|u|^{p}\right) d x-\frac{1}{p^{\star}} \int_{\mathbb{R}^{N}}\left(u^{+}\right)^{p^{\star}} d x-\lambda \int_{\mathbb{R}^{N}} F_{n}(x, u) d x
$$

for $u \in W^{1, p}\left(\mathbb{R}^{N}\right)$, where $W^{1, p}\left(\mathbb{R}^{N}\right)$ is the usual Sobolev space equipped with the norm

$$
\|u\|^{p}=\int_{\mathbb{R}^{N}}\left(|\nabla u|^{p}+|u|^{p}\right) d x .
$$

The functional $I_{\lambda, n}$ is well defined on $W^{1, p}\left(\mathbb{R}^{N}\right)$ and is of class $C^{1}$. Its Fréchet derivative is given by

$$
\begin{aligned}
\left\langle I_{\lambda, n}^{\prime}(u), v\right\rangle= & \int_{\mathbb{R}^{N}}\left(|\nabla u|^{p-2} \nabla u \nabla v+|u|^{p-2} u v\right) d x-\int_{\mathbb{R}^{N}}\left(u^{+}\right)^{p^{\star}-1} v d x \\
& -\lambda \int_{\mathbb{R}^{N}} f_{n}(x, u) v d x \quad \text { for } v \in W^{1, p}\left(\mathbb{R}^{N}\right) .
\end{aligned}
$$

Lemma 6.1. (i) Let $r_{1} \leq p$. Then there exist $\bar{\lambda}, \varrho, \alpha>0$ such that for every $0<\lambda<\bar{\lambda}$,

$$
I_{\lambda, n}(u) \geq \alpha \quad \text { for }\|u\|=\varrho,
$$

and each $n \in \mathbb{N}$.

(ii) If $p<r_{1}<p^{\star}$, then for each $\lambda>0$ there exist $\varrho, \alpha>0$ such that (16) holds for each $n \in \mathbb{N}$.

Proof. (i) It follows from $\left(f_{3}\right)$ and the Sobolev inequality that

$$
\begin{aligned}
& I_{\lambda, n}(u) \geq \frac{1}{p}\|u\|^{p}-\frac{1}{p^{\star}}\|u\|_{p^{\star}}^{p^{\star}}-\frac{\lambda}{r_{1}}\|b\|_{p^{\star} /\left(p^{\star}-r_{1}\right)}\|u\|_{p^{\star}}^{r_{1}}-\frac{\lambda c}{r_{2}}\|u\|_{r_{2}}^{r_{2}} \\
& \geq \frac{1}{p}\|u\|^{p}-\frac{1}{p^{\star} S^{p^{\star} / p}}\|u\|^{p^{\star}}-\frac{\lambda}{r_{1} S^{r_{1} / p}}\|b\|_{p^{\star} /\left(p^{\star}-r_{1}\right)}\|u\|^{r_{1}}-\frac{\lambda c}{r_{2} C_{r_{2}}^{r_{2}}}\|u\|^{r_{2}} \\
& =\|u\|^{p}\left(\frac{1}{p}-\frac{1}{p^{\star} S^{p^{\star} / p}}\|u\|^{p^{\star}-p}\right)-\lambda\left(\frac{\|b\|_{p^{\star} /\left(p^{\star}-r_{1}\right)}}{r_{1} S^{r_{1} / p}}\|u\|^{r_{1}}+\frac{c}{r_{2} C_{r_{2}}^{r_{2}}}\|u\|^{r_{2}}\right),
\end{aligned}
$$

where $C_{r_{2}}$ is the best Sobolev constant for the embedding of $W^{1, p}\left(\mathbb{R}^{N}\right)$ into $L^{r_{2}}\left(\mathbb{R}^{N}\right)$. First we choose constants $\varrho, \varrho_{1}>0$ so that

$$
\frac{1}{p}-\frac{1}{p^{\star} S^{p^{\star} / p}} \varrho^{p^{\star}-p}>\varrho_{1} .
$$

Then we choose a constant $\bar{\lambda}>0$ such that the inequality (16) holds for all $0<\lambda \leq \bar{\lambda}$ and some constant $\alpha>0$. 
(ii) If $p<r_{1}<p^{\star}$, then the above estimate takes the form

$$
I_{\lambda, n}(u) \geq\|u\|^{p}\left[\frac{1}{p}-\frac{1}{p^{\star} S^{p^{\star} / N}}\|u\|^{p^{\star}-p}-\lambda\left(C_{1}\|u\|^{r_{1}-p}+C_{2}\|u\|^{r_{2}-p}\right)\right]
$$

for some constants $C_{1}, C_{2}>0$ independent of $\lambda$ and $u$. From this estimate we easily deduce assertion (ii).

LEMma 6.2. In both cases (i) and (ii) of Lemma 6.1 there exist constants $0<\varrho_{1}<\varrho_{2}$ such that

$$
-\varrho_{2} \leq \inf _{\|u\| \leq \varrho} I_{\lambda, n}(u) \leq-\varrho_{1}
$$

for all $n \in \mathbb{N}$ sufficiently large.

Proof. Let $w \in C_{0}^{1}\left(\mathbb{R}^{N}\right), w \geq 0, w \not \equiv 0$, supp $w \subset \Omega$ and $\|w\| \leq \varrho$. Then for $t>0$,

$$
I_{\lambda, n}(t w) \leq \frac{t^{p}}{p} \int_{\mathbb{R}^{N}}\left(|\nabla w|^{p}+|w|^{p}\right) d x-\frac{t^{p^{\star}}}{p^{\star}} \int_{\mathbb{R}^{N}}|w|^{p^{\star}} d x-a t^{q} \int_{\mathbb{R}^{N}}|w|^{q} d x
$$

where $n$ is so large that $\operatorname{supp} w \subset B(0, n)$. Since $q<p$ we can choose $t>0$ small enough so that $I_{\lambda, n}(t w)<0$ and consequently the upper bound in (17) holds for $n$ sufficiently large. Finally, the lower bound in (17) follows from the estimates in the proof of Lemma 6.1.

7. Existence result for $\left(1_{\lambda, n}\right)$. For each $n \in \mathbb{N}$ we set

$$
c_{\lambda, n}=\inf _{\|u\| \leq \varrho} I_{\lambda, n}(u)
$$

According to Lemma 6.2 we have $-\varrho_{2} \leq c_{\lambda, n} \leq-\varrho_{1}$. Therefore we may assume that up to a subsequence $-\varrho_{2} \leq c_{\lambda}=\lim _{n \rightarrow \infty} c_{\lambda, n} \leq-\varrho_{1}$. For each $n \in \mathbb{N}$ there exists a sequence $\left\{u_{j}^{n}\right\} \subset B(0, \varrho)$ such that

$$
I_{\lambda, n}\left(u_{j}^{n}\right) \rightarrow c_{\lambda, n} \quad \text { and } \quad I_{\lambda, n}^{\prime}\left(u_{j}^{n}\right) \rightarrow 0 \quad \text { in } W^{-1, p^{\prime}}\left(\mathbb{R}^{N}\right)
$$

as $j \rightarrow \infty$, where $p^{\prime}=p /(p-1)$. Given $\varepsilon>0$ satisfying $c_{\lambda}+\varepsilon<0$ we can find $n_{0} \in \mathbb{N}$ such that $c_{\lambda, n} \in\left(c_{\lambda}-\varepsilon, c_{\lambda}+\varepsilon\right)$ for each $n \geq n_{0}$. Thus, for each $n \geq n_{0}$ we can select $u_{n}=u_{j_{n}}^{n}$ satisfying

$$
c_{\lambda}-\varepsilon<I_{\lambda, n}\left(u_{n}\right)<c_{\lambda}+\varepsilon \quad \text { and } \quad\left\|I_{\lambda, n}^{\prime}\left(u_{n}\right)\right\| \leq 1 / n .
$$

Since $\left\{u_{n}\right\}$ is bounded in $W^{1, p}\left(\mathbb{R}^{N}\right)$ we may assume that $u_{n} \rightarrow u$ in $W^{1, p}\left(\mathbb{R}^{N}\right)$.

We are now in a position to formulate the existence result for problem $\left(1_{\lambda, n}\right)$. We need the following assumption:

$\left(f_{4}\right) \quad$ There exist constants $p<\tau<p^{\star}$ and $1<\mu<p^{\star}$ such that

$$
\frac{1}{\tau} f(x, s) s-F(x, s) \geq c_{1}(x) s^{\mu}
$$

for all $x \in \mathbb{R}^{N}$ and $s \geq 0$, where $c_{1}$ is a function in $L^{p^{\star} /\left(p^{\star}-\mu\right)}\left(\mathbb{R}^{N}\right)$. 
The nonlinearity $f$ from the example in the paragraph following the assumption $\left(f_{3}\right)$ satisfies $\left(f_{4}\right)$ with $c_{1}(x)=(1 / \tau-1 / q) b(x), \mu=q$, provided $l<\tau<r_{2}$.

THEOREM 7.1. (i) If $r_{1} \leq p$, then there exists $\bar{\lambda}>0$ such that problem $\left(1_{\lambda}\right)$ has a nontrivial solution for each $0<\lambda<\bar{\lambda}$.

(ii) If $p<r_{1}<p^{\star}$, then problem $\left(1_{\lambda}\right)$ has a nontrivial solution for each $\lambda>0$.

Proof. It is sufficient to show that the weak limit of the sequence $\left\{u_{n}\right\}$ is not identically equal to 0 . The proof of this fact is similar to that of Theorem 1.1 in [16]. Therefore we only sketch it in some details.

Step 1. Applying P. L. Lions' concentration-compactness principle [15] we may assume that up to a subsequence

$$
\begin{gathered}
u_{n} \rightarrow u \quad \text { in } L_{\mathrm{loc}}^{s}\left(\mathbb{R}^{N}\right), 1 \leq s<p^{\star}, \quad u_{n}(x) \rightarrow u(x) \quad \text { a.e. on } \mathbb{R}^{N}, \\
\left|u_{n}\right|^{p^{\star}} \rightarrow \nu=|u|^{p^{\star}}+\sum_{i} \nu_{i} \delta_{x_{i}}+\nu_{\infty} \delta_{\infty}, \\
|\nabla u|^{p} \rightarrow \mu \quad \text { and } \quad \sum_{i} \nu_{i}^{p / p^{\star}}<\infty,
\end{gathered}
$$

where

$$
\nu_{\infty}=\lim _{R \rightarrow \infty} \limsup _{n \rightarrow \infty} \int_{|x| \geq R}\left|u_{n}\right|^{p^{\star}} d x .
$$

Step 2. In each bounded subset of $\mathbb{R}^{N}$ there are only a finite number of the points $x_{j}$. To establish this claim we use a family of smooth functions concentrating at $x_{j}$ and assumption $\left(f_{2}\right)$ and show as in [16] that $\mu\left(\left\{x_{i}\right\}\right) \leq$ $a_{R} \nu\left(\left\{x_{i}\right\}\right)=a_{R} \nu_{i}$. On the other hand we always have $\mu\left(\left\{x_{i}\right\}\right) \geq S \nu_{i}^{p / p^{\star}}$. Therefore $\nu_{i} \geq\left(S / a_{R}\right)^{N / p}$. Since $\sum_{i} \nu_{i}^{p / p^{\star}}<\infty$, we see that there are at most a finite number of $x_{j}$ in $B(0, r)$ for every $r>0$ and the claim easily follows.

Step 3. Using Step 2 one can show that $u_{n} \rightarrow u$ in $L^{p^{\star}}(K)$ for each compact set $K \subset \mathbb{R}^{N}-\bigcup\left\{x_{i}\right\}$. Indeed, as an immediate consequence of Step 2 we obtain $\int_{K}\left|u_{n}\right|^{p^{\star}} d x \rightarrow \int_{K}|u|^{p^{\star}} d x$ as $n \rightarrow \infty$. By the uniform convexity of the space $L^{p^{\star}}(K)$ we find that $u_{n} \rightarrow u$ in $L^{p^{\star}}(K)$. From this, using the fact that $\int_{\mathbb{R}^{N}}|\nabla u|^{p} d x$ and $\int_{\mathbb{R}^{N}}|u|^{p} d x$ are convex functionals, we deduce that $\nabla u_{n} \rightarrow \nabla u$ in $\left(L^{p}(K)\right)^{N}$ for each compact set $K \subset \mathbb{R}^{N}-\bigcup\left\{x_{i}\right\}$. This implies that $\nabla u_{n} \rightarrow \nabla u$ a.e. on $\mathbb{R}^{N}$. Applying Vitali's convergence theorem we show as in [16] that $u$ is a solution of $\left(1_{\lambda}\right)$ in the distributional sense. For details we refer to pages $9-10$ of [16]. It remains to show that 
$u \not \equiv 0$. Arguing indirectly assume that $u \equiv 0$. Thus, up to a subsequence

$$
\lim _{n \rightarrow \infty} \int_{\mathbb{R}^{N}}\left(\left|\nabla u_{n}\right|^{p}+\left|u_{n}\right|^{p}\right) d x=l>0,
$$

since otherwise $I_{\lambda, n}\left(u_{n}\right) \rightarrow 0$, which is impossible. By $\left(f_{4}\right)$ we have

$$
\begin{aligned}
0> & \lim _{n \rightarrow \infty}\left[I_{\lambda, n}\left(u_{n}\right)-\frac{1}{\tau}\left\langle I_{\lambda, n}^{\prime}\left(u_{n}\right), u_{n}\right\rangle\right] \\
\geq & \lim _{n \rightarrow \infty}\left[\left(\frac{1}{p}-\frac{1}{\tau}\right) \int_{\mathbb{R}^{N}}\left(\left|\nabla u_{n}\right|^{p}+\left|u_{n}\right|^{p}\right) d x+\left(\frac{1}{\tau}-\frac{1}{p^{\star}}\right) \int_{\mathbb{R}^{N}}\left(u_{n}^{+}\right)^{p^{\star}} d x\right. \\
& \left.+\int_{\mathbb{R}^{N}} c_{1}(x)\left|u_{n}\right|^{\mu} d x\right] \\
\geq & \left(\frac{1}{p}-\frac{1}{\tau}\right) l,
\end{aligned}
$$

which gives a contradiction.

8. Remark on the existence of a mountain-pass solution for problem $\left(1_{\lambda}\right)$. In the final section of this paper we indicate how to construct a mountain-pass solution for problem $\left(1_{\lambda}\right)$. This requires an additional assumption on the nonlinearity $f$ :

$\left(f_{5}\right) \quad$ There exists an open set $\Omega_{1} \subset \mathbb{R}^{N}$ such that

$$
F(x, s) \geq b s^{q_{1}}
$$

for every $x \in \Omega_{1}, s \geq 0$ and some constants $b>0$ and $1<q_{1}<p^{\star}$. Moreover, $p<q_{1}$ if $p^{2} \leq N$, and $q_{1}>p^{\star}-p /(p-1)$ if $p^{2}>N$.

This extra assumption in our model nonlinearity

$$
f(x)=b(x) s^{q-1}-d(x) s^{l-1}+C s^{r_{2}-1}
$$

is satisfied provided $d(x)=0$ for $x \in B(0, R)$ with $q_{1}=r_{2}$ and $r_{2}$ satisfying the conditions for $q_{1}$ from $\left(f_{5}\right)$.

We recall that the best Sobolev constant $S$ in $W^{1, p}\left(\mathbb{R}^{N}\right)$ is defined by

$$
S=\inf _{u \in \mathcal{D}^{1, p}\left(\mathbb{R}^{N}\right)-\{0\}} \frac{\int_{\mathbb{R}^{N}}|\nabla u|^{p} d x}{\left(\int_{\mathbb{R}^{N}}|u|^{p^{\star}} d x\right)^{p / p^{\star}}} .
$$

It is well known [10] that the infimum in (18) is achieved by a family of functions

$$
W_{\varepsilon}(x)=\frac{c_{N} \varepsilon^{(N-p) / p^{2}}}{\left(\varepsilon+|x|^{p /(p-1)}\right)^{(N-p) / p}}, \quad \varepsilon>0,
$$


for $x \in \mathbb{R}^{N}$, where

$$
c_{N}=\left\{N\left[\frac{N-p}{p-1}\right]^{p-1}\right\}^{(N-p) / p^{2}} .
$$

We also have $\int_{\mathbb{R}^{N}}\left|\nabla W_{\varepsilon}\right|^{p} d x=\int_{\mathbb{R}^{N}} W_{\varepsilon}^{p^{\star}} d x=S^{N / p}$.

Theorem 8.1. Suppose that assumptions $\left(f_{1}\right), \ldots,\left(f_{5}\right)$ hold. Then in both cases (i) and (ii) of Theorem 7.1 problem $\left(1_{\lambda}\right)$ admits a mountain-pass solution. (This means that in both cases (i) and (ii) problem $\left(1_{\lambda}\right)$ has at least two distinct solutions.)

Proof. We follow the ideas from [16] (see the proof of Theorem 1.1 there).

Step I. We use the estimate from the proof of Lemma 6.2 to obtain

$$
I_{\lambda, n}(t w) \leq \frac{t^{p}}{p} \int_{\mathbb{R}^{N}}\left(|\nabla w|^{p}+|w|^{p}\right) d x-\frac{t^{p^{\star}}}{p^{\star}} \int_{\mathbb{R}^{N}}|w|^{p^{\star}} d x-a t^{q} \int_{\mathbb{R}^{N}}|w|^{q} d x .
$$

However, this time for sufficiently large $t>0$ we get $I_{\lambda, n}(t w)<0$. Combining this with Lemma 6.1 we see that in both cases (i) and (ii), $I_{\lambda, n}$ satisfies (uniformly in $n$ ) the conditions of the geometry of the mountain-pass theorem.

Step II. Let $x_{0} \in \Omega_{1}$ and choose $r>0$ so that $B\left(x_{0}, 2 r\right) \subset \Omega_{1}$. We assume that $B\left(x_{0}, 2 r\right) \subset B(0, n)$ for all $n \geq n_{0}$ for some integer $n_{0}$. We define a function $\varphi \in C_{0}^{1}\left(\mathbb{R}^{N}\right)$ such that $\varphi(x)=1$ on $B\left(x_{0}, r\right), \varphi(x)=0$ on $\mathbb{R}^{N}$ $B\left(x_{0}, 2 r\right)$ and $0 \leq \varphi(x) \leq 1$ on $\mathbb{R}^{N}$ and set $v_{\varepsilon}=\varphi W_{\varepsilon}\left(\cdot-x_{0}\right) /\left\|\varphi W_{\varepsilon}\left(\cdot-x_{0}\right)\right\|_{p^{\star}}$. We claim that there exist $\varepsilon, d_{\lambda}>0$ such that

$$
\max _{t \geq 0} I_{\lambda, n}\left(t v_{\varepsilon}\right) \leq d_{\lambda}<\frac{S^{N / p}}{N}
$$

for $n \geq n_{0}$. Setting $Y_{\varepsilon}=\int_{\mathbb{R}^{N}}\left(\left|\nabla v_{\varepsilon}\right|^{p}+\left|v_{\varepsilon}\right|^{p}\right) d x$, we derive from assumption $\left(f_{4}\right)$ that

$$
\begin{aligned}
I_{\lambda, n}\left(t v_{\varepsilon}\right) & =\frac{t^{p} Y_{\varepsilon}}{p}-\frac{t^{p^{\star}}}{p^{\star}}-\lambda \int_{\mathbb{R}^{N}} F_{n}\left(x, t v_{\varepsilon}\right) d x \\
& \leq \frac{t^{p} Y_{\varepsilon}}{p}-\frac{t^{p^{\star}}}{p^{\star}}-\lambda t^{q_{1}} b \int_{\mathbb{R}^{N}}\left|v_{\varepsilon}\right|^{q_{1}} d x .
\end{aligned}
$$

Let

$$
J_{\lambda}\left(t v_{\varepsilon}\right)=\frac{t^{p} Y_{\varepsilon}}{p}-\frac{t^{p^{\star}}}{p^{\star}}-\lambda t^{q_{1}} b \int_{\mathbb{R}^{N}}\left|v_{\varepsilon}\right|^{q_{1}} d x .
$$

It is sufficient to show that there exist $d_{\lambda}, \varepsilon>0$ such that

$$
\max _{t \geq 0} J_{\lambda}\left(t v_{\varepsilon}\right) \leq d_{\lambda}<\frac{S^{N / p}}{N} .
$$


For each $\varepsilon>0$ there exists $t_{\varepsilon}>0$ such that

$$
\max _{t \geq 0} J_{\lambda}\left(t v_{\varepsilon}\right)=J_{\lambda}\left(t_{\varepsilon} v_{\varepsilon}\right)
$$

with $0<t_{\varepsilon} \leq Y_{\varepsilon}^{1 /(p-1)}$. Consequently, we have

$$
J_{\lambda}\left(t_{\varepsilon} v_{\varepsilon}\right) \leq \frac{1}{N} Y_{\varepsilon}^{N / p}-\lambda b_{1} \int_{\mathbb{R}^{N}}\left|v_{\varepsilon}\right|^{q-1} d x
$$

for some constant $b_{1}>0$. Suppose that $p^{2} \leq N$. Using the explicit form of $W_{\varepsilon}$ we easily derive the following estimate:

$$
\begin{aligned}
J_{\lambda}\left(t_{\varepsilon} v_{\varepsilon}\right) & \leq \frac{S^{N / p}}{N}+O\left(\varepsilon^{(N-p) / p}\right)+O\left(\varepsilon^{p-1}\right)-\lambda b_{1} \int_{\mathbb{R}^{N}}\left|v_{\varepsilon}\right|^{q_{1}} d x \\
& =\frac{S^{N / p}}{p}+O\left(\varepsilon^{p-1}\right)-\lambda b_{1} \int_{\mathbb{R}^{N}}\left|v_{\varepsilon}\right|^{q_{1}} d x .
\end{aligned}
$$

The term $O\left(\varepsilon^{p-1}\right)$ is due to the integral $\int_{\mathbb{R}^{N}}\left|v_{\varepsilon}\right|^{p} d x$ appearing in $Y_{\varepsilon}$. Thus, there exists $M>0$ such that

$$
J_{\lambda}\left(t_{\varepsilon} v_{\varepsilon}\right) \leq \frac{S^{N / p}}{N}+\varepsilon^{p-1}\left(M-\frac{\lambda b_{1}}{\varepsilon^{p-1}} \int_{\mathbb{R}^{N}}\left|v_{\varepsilon}\right|^{q_{1}} d x\right) .
$$

Arguing as in the proof of Proposition 5.1 in [16] we derive from this the estimate

$$
J_{\lambda}\left(t_{\varepsilon} v_{\varepsilon}\right) \leq \frac{S^{N / p}}{N}+\varepsilon^{p-1}\left(M-\lambda b_{2} \varepsilon^{\left[\left(\frac{N-p}{p^{2}}-\frac{N-p}{p}\right) q_{1}+\frac{(p-1) N}{p}+1-p\right]}\right)
$$

for some constant $b_{2}>0$. Since

$$
\left[\frac{N-p}{p^{2}}-\frac{N-p}{p}\right] q_{1}+(p-1)\left(\frac{N}{p}-1\right)<0
$$

is equivalent to $p<q_{1}$, our claim follows from the estimate (20). In a similar manner we consider the case $p^{2}>N$.

Step III. We now apply the mountain-pass theorem to the functionals $I_{\lambda, n}$ for every $n \geq n_{0}$ to obtain the mountain-pass levels $c_{\lambda, n}$ and PalaisSmale sequences $\left\{u_{j}^{n}\right\}$ in $W^{1, p}\left(\mathbb{R}^{N}\right)$ satisfying

$$
I_{\lambda, n}\left(u_{j}^{n}\right) \rightarrow c_{\lambda, n} \quad \text { and } \quad I_{\lambda_{n}}^{\prime}\left(u_{j}^{n}\right) \rightarrow 0 \quad \text { in } W^{-1, p^{\prime}}\left(\mathbb{R}^{N}\right)
$$

as $j \rightarrow \infty$. We then choose a subsequence $u_{n}=u_{j_{n}}^{n}$ satisfying

$$
c_{\lambda}-\varepsilon \leq I_{\lambda, n}\left(u_{n}\right) \leq c_{\lambda}+\varepsilon, \quad\left\|I_{\lambda, n}^{\prime}\left(u_{n}\right)\right\| \leq 1 / n
$$

for $n \geq n_{0}$ and some $\varepsilon>0$, where up to a subsequence $\lim _{n \rightarrow \infty} c_{\lambda, n}=c_{\lambda}$. Arguing as in Lemma 6.1 of [16] we show that $u_{n} \rightarrow u \not \equiv 0$ in $W^{1, p}\left(\mathbb{R}^{N}\right)$ and $u$ is a solution of $\left(1_{\lambda}\right)$. 


\section{References}

[1] C. O. Alves, J. V. Gonçalves and O. H. Miyagaki, On elliptic equations in $\mathbb{R}^{N}$ with critical exponents, Electron. J. Differential Equations 1996, no. 09, 1-11.

[2] A. Ambrosetti, J. García Azorero and I. Peral, Elliptic variational problems in $\mathbb{R}^{N}$ with critical growth, J. Differential Equations 108 (2000), 10-32.

[3] A. Ambrosetti and P. Rabinowitz, Dual variational methods in critical point theory and applications, J. Funct. Anal. 14 (1976), 349-381.

[4] T. Bartsch, Infinitely many solutions of a symmetric Dirichlet problem, Nonlinear Anal. 20 (1993), 1205-1216.

[5] T. Bartsch and M. Willem, On an elliptic equation with concave and convex nonlinearities, Proc. Amer. Math. Soc. 123 (1995), 3555-3361.

[6] V. Benci and G. Cerami, Existence of positive solutions of the equation $-\Delta u+$ $a(x) u=u^{(N+2) /(N-2)}$ in $\mathbb{R}^{N}$, J. Funct. Anal. 88 (1990), 90-117.

[7] A. K. Ben-Naoum, C. Troestler and M. Willem, Extrema problem with critical Sobolev exponents on unbounded domains, Nonlinear Anal. 26 (1996), 823-833.

[8] J. Chabrowski, Concentration-compactness principle at infinity and semilinear elliptic equations involving critical and subcritical Sobolev exponents, Calc. Var. 3 (1995), 493-512.

[9] J. Chabrowski and J. F. Yang, Multiple semiclassical solutions of the Schrödinger equation involving a critical Sobolev exponent, Portugal. Math. 57 (2000), 273-284.

[10] H. Egnell, Existence and nonexistence results for $m$-Laplace equations involving critical Sobolev exponents, Arch. Rational Mech. Anal. 104 (1988), 57-77.

[11] I. Ekeland, On the variational principle, J. Math. Anal. Appl. 47 (1974), 324-353.

[12] J. García Azorero, E. Montefusco and I. Peral, Bifurcation for the p-Laplacian in $\mathbb{R}^{N}$, Adv. Differential Equations 5 (2000), 435-464.

[13] J. V. Gonçalves and C. O. Alves, Existence of positive solutions for m-Laplacian equations in $\mathbb{R}^{N}$ involving critical Sobolev exponents, Nonlinear Anal. 32 (1998), $53-70$.

[14] J. V. Gonçalves and H. Miyagaki, Multiple positive solutions for semilinear elliptic equations in $\mathbb{R}^{N}$ involving subcritical exponents, ibid. 32 (1998), 41-51.

[15] P. L. Lions, The concentration-compactness principle in the calculus of variations, The limit case, Rev. Mat. Iberoamericana 1 (1985), no. 1, 145-201 and no. 2, 45-120.

[16] E. A. de B. e Silva and S. H. M. Soares, Quasilinear Dirichlet problems in $\mathbb{R}^{N}$ with critical growth, Nonlinear Anal. 43 (2001), 1-20.

[17] G. Talenti, Best constant in Sobolev inequality, Ann. Mat. Pura Appl. 101 (1976), 353-372.

[18] S. B. Tshinanga, On multiple solutions of semilinear elliptic equation on unbounded domains with concave and convex nonlinearities, Nonlinear Anal. 28 (1997), 809814.

Department of Mathematics The University of Queensland St. Lucia 4072, Qld, Australia E-mail: jhc@maths.uq.edu.au
Centre of Applied Mathematics University of West Bohemia Univerzitni 22, 30614 Plzen, Czech Republic E-mail: pdrabek@kma.zcu.cz 